\title{
A DIC Based Technique to Measure the Contraction of a Skeletal Muscle Engineered Tissue
}

\author{
Emanuele Rizzuto, ${ }^{1}$ Silvia Carosio, ${ }^{2}$ Martina Faraldi, ${ }^{3}$ Simona Pisu, ${ }^{3}$ \\ Antonio Musarò, ${ }^{2,3}$ and Zaccaria Del Prete ${ }^{1,2}$ \\ ${ }^{1}$ Department of Mechanical and Aerospace Engineering, University of Rome La Sapienza, Via Eudossiana 18, 00184 Rome, Italy \\ ${ }^{2}$ Center for Life Nano Science@Sapienza, Istituto Italiano di Tecnologia, Viale Regina Elena 291, 00161 Rome, Italy \\ ${ }^{3}$ Institute Pasteur Cenci-Bolognetti, Interuniversity Institute of Myology, Department of Anatomical, Histological, \\ Forensic Medicine and Orthopedic Sciences (DAHFMO), Unit of Histology and Medical Embryology, University of Rome La Sapienza, \\ Via A. Scarpa 16, 00161 Rome, Italy
}

Correspondence should be addressed to Emanuele Rizzuto; emanuele.rizzuto@uniromal.it

Received 13 November 2015; Revised 11 February 2016; Accepted 15 February 2016

Academic Editor: Hoon Eui Jeong

Copyright (C) 2016 Emanuele Rizzuto et al. This is an open access article distributed under the Creative Commons Attribution License, which permits unrestricted use, distribution, and reproduction in any medium, provided the original work is properly cited.

\begin{abstract}
Tissue engineering is a multidisciplinary science based on the application of engineering approaches to biologic tissue formation. Engineered tissue internal organization represents a key aspect to increase biofunctionality before transplant and, as regarding skeletal muscles, the potential of generating contractile forces is dependent on the internal fiber organization and is reflected by some macroscopic parameters, such as the spontaneous contraction. Here we propose the application of digital image correlation (DIC) as an independent tool for an accurate and noninvasive measurement of engineered muscle tissue spontaneous contraction. To validate the proposed technique we referred to the X-MET, a promising 3-dimensional model of skeletal muscle. The images acquired through a high speed camera were correlated with a custom-made algorithm and the longitudinal strain predictions were employed for measuring the spontaneous contraction. The spontaneous contraction reference values were obtained by studying the force response. The relative error between the spontaneous contraction frequencies computed in both ways was always lower than $0.15 \%$. In conclusion, the use of a DIC based system allows for an accurate and noninvasive measurement of biological tissues' spontaneous contraction, in addition to the measurement of tissue strain field on any desired region of interest during electrical stimulation.
\end{abstract}

\section{Introduction}

Tissue engineering represents a multidisciplinary approach aimed at "producing" ex vivo living tissues [1] on the basis of three fundamental tools: cells, scaffolds, and growth factors. The application of engineering techniques to biological issues ranges from the nanometric scale, that affects the cell phenotype, to the micro and macro features, that influence nutrient flows, and could make significant contributions to the realization of biofunctional and biocompatible 3dimensional tissues. The ultimate goal of tissue engineering is the regeneration of patients' own tissues and organs with high biocompatibility and biofunctionality and without immune rejection typical of inorganic biomaterials. However, despite the remarkable advances recently obtained, artificial tissues and organs still need a biocompatibility and biofunctionality improvement [2].

Within this context, we were called to respond to the need of measuring ex vivo engineered skeletal muscle contractile properties. Skeletal muscle is a very complex tissue, and ex vivo engineered tissues gained more and more importance [3-7] because of their potential to be used for injured and infarcted patients. To monitor engineered muscle tissue growth we recently proposed [8] the application of Digital Image Correlation (DIC) in combination with standard force measurement. DIC is a versatile methodology deriving 
from industrial and civil engineering $[9,10]$ that presents several advantages also for applications with biological tissues [11-13], such as the absence of contact with the specimen, the possibility to use the natural texture as the correlation pattern, and the opportunity to measure strains on a specific region of interest (ROI). The use of this technique allowed pinpointing the tissue nonhomogeneous zones, returning useful information for improving the tissue generation process. The growth process improvement and the optimization of the techniques, in fact, represent the first steps on the road map of three generations of the regenerative medicine industry [14], and the final aim of this first generation industry is to increase the tissue functionality before transplant [15].

The spontaneous contractile activity of cultured skeletal muscle cells was first reported in 1915 [16] and still represents a crucial parameter of contractile tissues $[17,18]$. The contractile properties of engineered muscle tissues, and in particular the spontaneous contraction, strongly depend on their internal functional organization. Tissue-engineered muscles should reproduce the native fibers organization: both in-series and in-parallel connections of short myofibers to the surrounding matrix and to other fibers, in fact, significantly contribute to the total force generation [19].

The ionic conductance responsible for the origin and modulation of muscle cell spontaneous activity is normally examined using the whole-cell patch-clamp technique [20, 21]. This methodology cannot be applied to 3D skeletal muscle engineered tissues and, to date, the unique technique for studying the contractile activity of $3 \mathrm{D}$ tissues is based on the force response measurement obtained using microforce transducers $[3,4,22]$. The development of new approaches to measure biological tissues' contractile properties is therefore of high interest, and noninvasive techniques are always more attractive $[23,24]$. A contactless automated approach to measure this distinguished feature could therefore allow avoiding time consuming, incomplete and subjective measurements [25].

In view of this, here we propose the use of Digital Image Correlation as a tool for performing accurate and noninvasive measurement of ex vivo skeletal muscle tissue spontaneous contraction. The spontaneous contraction values of X-MET, a promising 3-dimensional ex vivo engineered muscle tissue [3], were obtained analyzing the longitudinal strain predictions returning from image correlation and were compared with the values gathered through the standard force measurement. In fact, even if the outcomes of these two techniques pertain different variables (strain and force, resp.), both of them follow the contractile behavior of the tissue, thereby allowing determining the tissue contraction frequency. Because skeletal muscle engineered tissues can be subjected to considerable dynamics, a high speed camera was employed and, to have a comprehensive investigation, we tested the specimens with two different acquisition speeds. Should the comparison between the traditional technique and the Digital Image Correlation return convincing results, the use of DIC would allow performing repetitive and noninvasive measurements of engineered muscle tissues to monitor their growth. The use of force transducers, in fact, would imply a recurring stress on the tissue, by attaching and

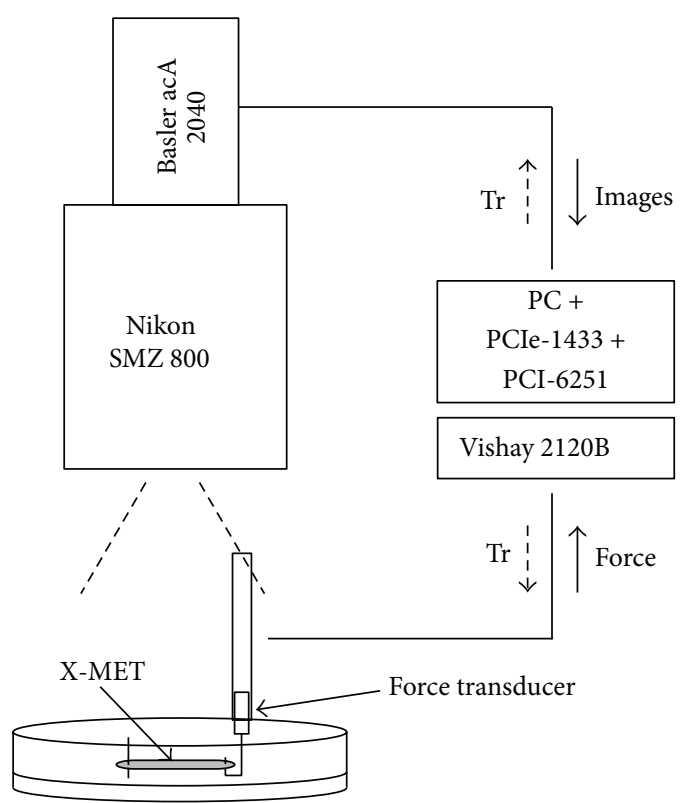

FIGURE 1: Experimental set-up. Main instruments and data flows of the experimental system. The personal computer (PC) is equipped with a data acquisition board (PCI-6251) and a frame grabber (PCIe1433), triggering ( $\mathrm{Tr}$ ) both image and force data acquisition.

detaching it from the transducer each time a measurement has to be done.

Finally, to have a comprehensive evaluation of the role that DIC can have in the measurement of engineered muscle tissues' contractile properties we also studied the strain predictions when stimulating the X-MET with a pulse train at tetanic frequency. Of note, X-MET is obtained from murine primary cultures of skeletal muscle tissue and shows the presence of various cellular populations such as myoblasts, fibroblasts, and endothelial cells, which guarantees a cellular complexity similar to that of the original muscle tissue. Thus, ex vivo engineered tissue can be placed in the high level of biological complexity of the regenerative medicine road map [14].

\section{Materials and Methods}

2.1. Experimental Set-Up. All the experiments were conducted within the animal welfare regulations and guidelines of the Italian national law D.L. 04/03/2014, n.26, about the use of animals for research. The X-MET specimens employed to test the proposed technique were obtained from skeletal muscle primary cultures of C57BL/6 mice (14-18 weeks) according to the protocol illustrated by Carosio et al. [3].

As depicted in Figure 1, the specimen to be tested was placed in a silicon coated dish and mounted between a fixed pin and a microforce transducer (Kronex AE801) for isometric measurements. For the entire duration of the experiment the tissue was kept soaked in its culturing medium and maintained at the desired temperature of $37^{\circ} \mathrm{C}$ with the use of a temperature controlled plate (Okolab s.r.l., H401). Images were gathered through the use of 
a high frequency camera (Basler acA2040-180 km) mounted on a stereomicroscope (NIKON SMZ 800), while force measurements were performed connecting the microforce transducer to a strain gauge conditioner (VISHAY 2120B module) for bridge completion. At the magnification level of $6.3 \mathrm{x}$, the resulting images have a rectangular size of $1.94 \mathrm{~mm} \times$ $0.57 \mathrm{~mm}$, thus allowing acquiring about $1 / 5$ of the entire tissue length [3]. The suitable lighting was provided by a diascopic halogen $30 \mathrm{~W}$ lamp. The camera employed in this work has a maximum frame rate of $180 \mathrm{fps}$ at the full resolution of $2048 \times 2048$ pixels. To increase the speed of acquisition up to values higher than $400 \mathrm{fps}$ we selected both a horizontal and a vertical binning, thus reducing the resolution at 1024 $\times 1024$ pixels. Of note, this binning procedure was also necessary to enhance the amount of light available at the sensor. Fast image acquisition and optical magnification, in fact, are two factors that reduce the light intensity, thus compromising the functionality of the postprocessing correlation algorithm. Finally, because of the uniaxial shape of the tissue specimens to be tested, the vertical resolution was further reduced. At the selected resolution of $1024 \times 300$ pixels, the maximum speed of acquisition was $620 \mathrm{fps}$. Nonetheless, all the experimental tests were performed acquiring the images at lower frequencies, because of the limitation due to light intensity. In particular, two frequencies of acquisition were tested, $400 \mathrm{fps}$, which implies an exposure time of $2400 \mu \mathrm{s}$, and $500 \mathrm{fps}$, with an exposure time of $1900 \mu \mathrm{s}$. On the other hand, for all the tests the force acquisition frequency was set at $2200 \mathrm{~Hz}$. The tests performed to evaluate the role of DIC in the characterization of engineered muscle tissue contractility during tetanic stimulation employed a dedicated pulse stimulator (Aurora Scientific Inc. 701B). Two platinum electrodes were located parallel to the specimen allowing stimulating the tissue by means of the physiological solution. A computer equipped with a data acquisition board (DAQ NI-PCI 6251M) and a frame grabber (NI PCIe1433) was programmed with LabView 2011 software. The custom-made program allowed accurately synchronizing the beginning of force and image data flows acquisition and, where necessary, of pulse stimulation.

The acquired images then were processed with an original correlation algorithm [26] to compute the 2D surface strain field, but only the longitudinal deformation was used to gather the tissue spontaneous contraction. Spontaneous contraction reference values, in fact, were obtained from force measurements performed measuring the tissue's response along the longitudinal axis. The specific forces were computed by dividing the acquired force values by the X-MET crosssectional area as in Carosio et al. [3]. The algorithm we employed to obtain the strain predictions was originally developed by Broggiato et al. [26] to measure thin metal sheets' strain field and was optimized to work with small biological tissue samples subjected to controlled lengthening [13] and with ex vivo engineered tissues electrically stimulated [8]. Of note, for all the measurements we performed the irregular surface pattern of X-METs was taken as the reference speckle for the correlation algorithm, thus avoiding strewing the tissue with artificial powders that could induce alterations in the vitality.

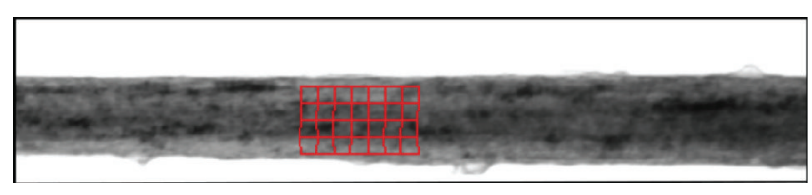

(a)

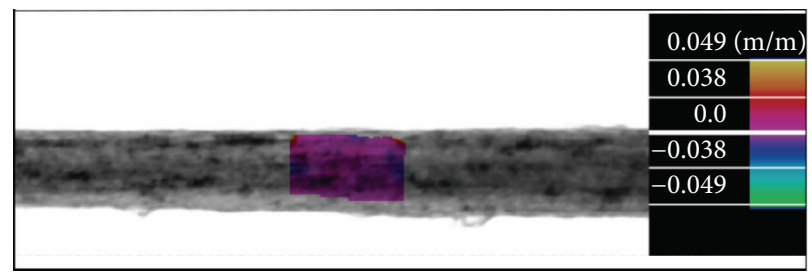

(b)

FIgURE 2: ROI selection. Example of a region of interest (ROI) superimposed on an X-MET surface (a) for longitudinal strain predictions (b).

2.2. Spontaneous Contraction Measurement. To validate our DIC based system as a tool for measuring skeletal muscle engineered tissues' spontaneous contraction, we tested $4 \mathrm{X}$ METs with a synchronous acquisition of both force and images. The specimen to be tested was focused close to the fixed pin, and the image acquisition lasted for at least $2 \mathrm{sec}-$ onds. To find out the acquisition parameters that guaranteed the most accurate results, two different speeds of acquisition were tested, $400 \mathrm{fps}$ and $500 \mathrm{fps}$. Images were then correlated as described above, and the average longitudinal strain value of all the nodes included in the selected ROI was used as representative of the tissue's contraction. For all the tests, the ROI was randomly chosen so to be rectangular, with the long side parallel to the longitudinal axis, and was made up of at least 20 nodes, as shown, for example, in Figure 2.

For both force response and strain data, the spontaneous contraction frequency was computed through the Fast Fourier Transform (FFT). The use of the FFT allowed finding the main signal component that characterizes the tissue's contraction, reducing the noise influence. For our tests, in fact, the noise is usually represented by very small peaks at frequencies considerably higher than the one of interest. Of note, since our aim was to devise a general method for measuring the tissue spontaneous contraction in a fast but accurate manner, for all the tests we performed we took into account only the highest peak that each FFT analysis returned. The spontaneous contraction frequencies obtained from the force and strain response were then compared, and the relative error was employed to establish the DIC system accuracy according to the simple equation: $\left(f_{s}-f_{f}\right) / f_{f} \%$, where $f_{s}$ is the frequency value obtained by the DIC strain predictions and $f_{f}$ is the value returned analyzing the force signal.

2.3. Statistical Analysis. Differences in the spontaneous contraction frequency values obtained analyzing the strain predictions of the DIC algorithm and the force response measurements, as well as differences in the relative error 


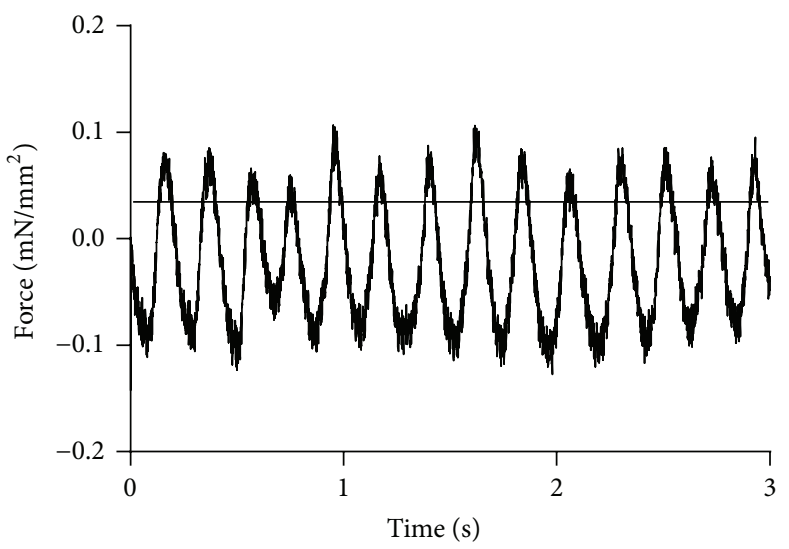

(a)

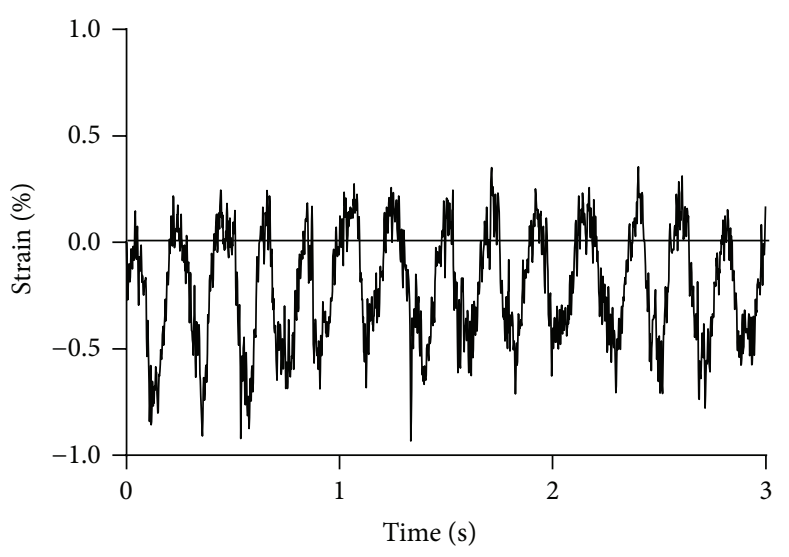

(c)

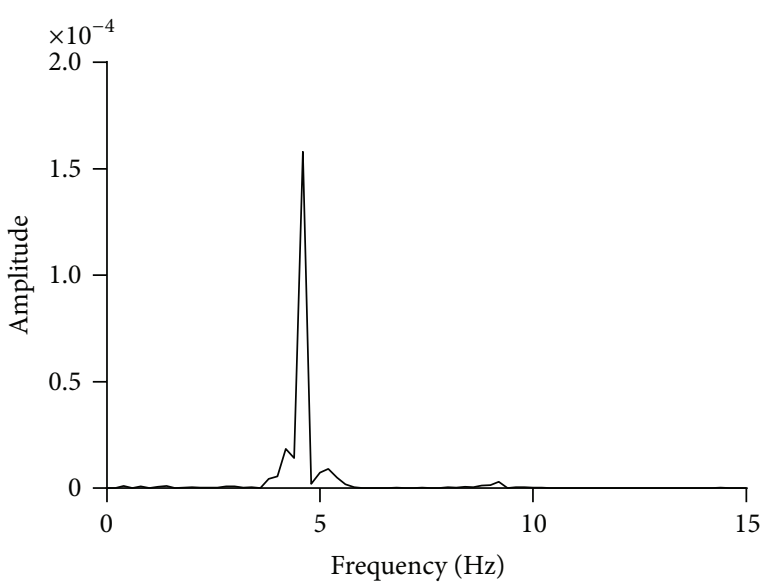

(b)

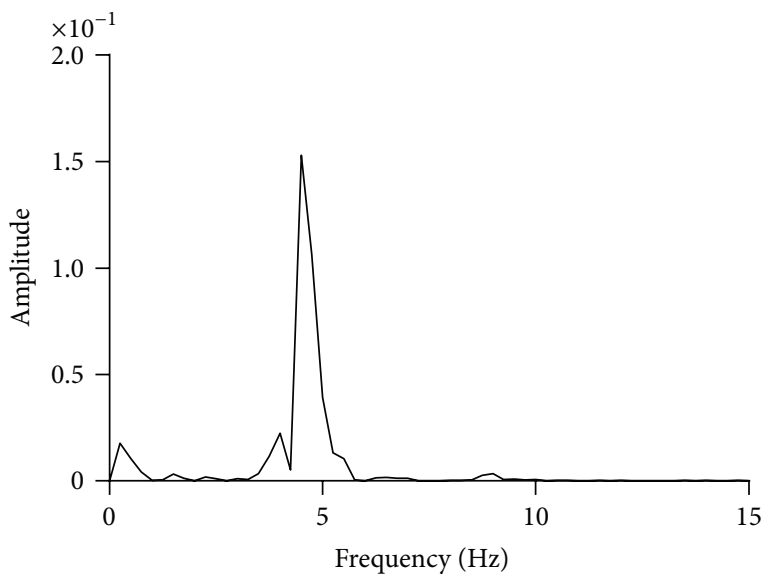

(d)

Figure 3: Data analysis. Example of an X-MET spontaneous contraction measured through the micro-force transducer (a), with the corresponding FFT analysis (b), and through the digital image correlation technique (c), again with the corresponding FFT analysis (d). The values in (b) and (d) are $N_{\mathrm{RMS}}^{2}$ and $(\mathrm{m} / \mathrm{m})_{\mathrm{RMS}}^{2}$ respectively. Images were acquired at $500 \mathrm{fps}$.

computed at $400 \mathrm{fps}$ and $500 \mathrm{fps}$, were evaluated with the nonparametric Student's $t$-test and considered significant when $p<0.05$.

\section{Experimental Results and Discussion}

Tissue spontaneous contraction was calculated referring to the force response and to the acquired images as described in Materials and Methods, Section 2.2, and an example of the results is reported in Figure 3 for a test performed at $500 \mathrm{fps}$.

Figures 3(a) and 3(b) show the force signal and the resulting FFT analysis, while Figures 3(c) and 3(d) show the strain predictions for the selected ROI computed through the DIC algorithm with the corresponding FFT outcome. The comparison between force (Figure 3(a)) and strain (Figure 3(c)) responses, that represent the outcomes obtained in the traditional method and through the DIC technique, respectively, allowed verifying that both signals adequately stand for the pulsating behavior the tissue undergoes when kept fixed in isometric conditions within its culturing medium. Of note, although the two techniques measure different variables, namely, force and strain, both of them allow obtaining the spontaneous contraction frequency of the muscle tissue. In addition, even if both signals show the presence of noise, the FFT outcomes (Figures 3(b) and 3(d)) confirmed that this mathematical procedure is suitable to pinpoint the main component of the beating frequency, almost nullifying the noise effect.

A combined analysis of force (Figure 3(a)) and strain responses (Figure 3(c)) shows an opposition of phase between the two traces, highlighting that when the tissue generates a contractile force, the ROI of the sample we choose (close to the fixed pin) is truly shortening. At this point, it has to be noted that all our tests were performed in isometric conditions, thus entailing a global null longitudinal strain of the specimen. Given that we measured a negative strain, it is then feasible that the central part of the tissue underwent a positive strain to compensate for this small shortening. Of note, the software we developed to control the experiments subtracted the force offset before the beginning of the acquisition; in view of this, the fact that the force response immediately decreased to values lower than zero 
TABLE 1: X-MET's spontaneous contraction. Mean \pm SD of X-MET's spontaneous contraction measured using the force response with reference to the acquired images, at both $400 \mathrm{fps}$ and $500 \mathrm{fps} . n=4$.

\begin{tabular}{lcccc}
\hline & From force at $400 \mathrm{fps}$ & From strain at $400 \mathrm{fps}$ & From force at $500 \mathrm{fps}$ & From strain at $500 \mathrm{fps}$ \\
\hline Mean $\pm \mathrm{SD}(\mathrm{Hz})$ & $4.520 \pm 0.404$ & $4.523 \pm 0.403$ & $4.545 \pm 0.592$ & $4.546 \pm 0.591$ \\
\hline
\end{tabular}

only means that the tissue was in a relaxing phase of its contraction. Accordingly, the initial strain is increasing.

Table 1 shows the spontaneous frequency values measured through the force response and the DIC predictions for both $400 \mathrm{fps}$ and $500 \mathrm{fps}$ acquisition speeds. The statistical analysis performed comparing the force group with the strain group, once for $400 \mathrm{fps}$ and once for $500 \mathrm{fps}$, confirmed the null hypothesis, thus indicating that the groups can be considered as originating from the same populations. Notably, the X-MET spontaneous contraction values here obtained are different from the values previously reported [3]. This difference was expected and is related to the different medium employed during the experimental tests. In our previous paper, in fact, we performed measurements that involved electrical stimulation of the tissue, thus requiring the medium to be free of calcium ions. Here, on the contrary, we are proposing a method for monitoring the muscle engineered tissue during its growth; therefore the tests were performed within their culturing medium, very enriched in nutrients. The average spontaneous contraction frequencies values obtained here are, in fact, higher than the value previously reported.

Despite the fact that the tested groups have an average mean that is not statistically different among each other, to estimate the accuracy of the proposed method and to find out whether an increase in the speed of acquisition would increase the measurement accuracy, we computed the relative error between the spontaneous contraction values obtained through the DIC predictions and the reference values obtained from the tissue's force response. Figure 4 shows the relative error values obtained at $400 \mathrm{fps}$ and $500 \mathrm{fps}$. Experimental results pointed out a high accuracy of the methodology for both acquisition speeds. The relative error, in fact, is lower than $0.15 \%$ for all the experiments we performed. Nonetheless, a lower mean error, although not statistically significant, as well as a lower standard deviation, was reported when increasing the speed of acquisition from $400 \mathrm{fps}$ to $500 \mathrm{fps}$. This outcome suggests that when long acquisition times are required, a $400 \mathrm{fps}$ acquisition frequency can be utilized, while, on the contrary, when a higher accuracy is necessary, the acquisition speed can be increased up to $500 \mathrm{fps}$. In the latter case, to avoid overloading the system memory, shorter acquisitions are advisable.

Finally, it has to be noted that the proposed system showed also good sensitivity values. The accuracy values reported in Figure 4, in fact, were obtained when measuring the spontaneous contraction on strain signals of only about $1 \%$ peak to peak amplitude, as in the example reported in Figure 3(c).

Figure 5 shows an example of the outcome obtained stimulating an X-MET at tetanic frequency $(60 \mathrm{~Hz})$ and simultaneously measuring force (Figure 5(a)) and strain (Figures 5(a)

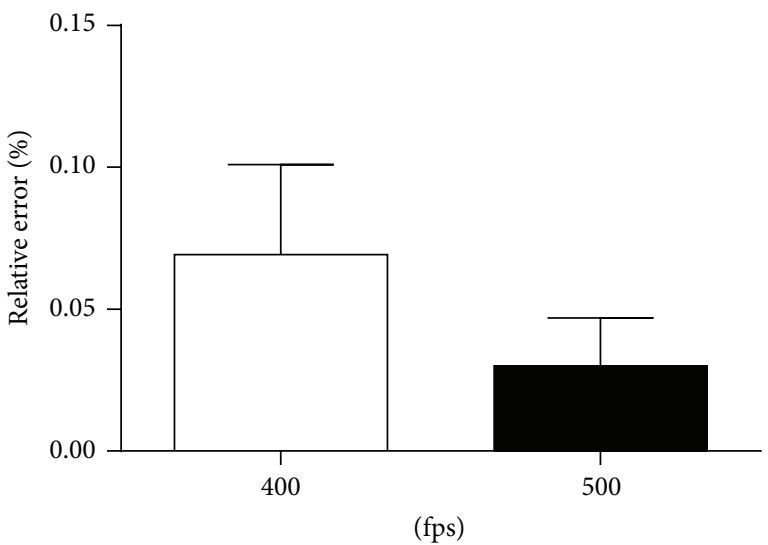

FIgURE 4: Relative error. Mean \pm SEM of the relative error between the spontaneous contraction values measured through the tissue force response and the longitudinal strain behavior at $400 \mathrm{fps}$ (white) and at $500 \mathrm{fps}$ (black). $n=4$.

and 5(b)). As indicated by Carosio et al. [3] for the X-MET the tetanic force increased with increasing frequency up to $70 \mathrm{~Hz}$ but, on average, the asymptotic value is already achieved at $60 \mathrm{~Hz}$. In particular, the tissue strain fields were measured once close to the fixed pin (Figure 5(b)) and once in the center (Figure 5(c)) of the specimen. Again, both the force and strain responses are in complete agreement and are coherent with the electrical stimulation paradigm. Contrary to the spontaneous contraction, during the electrical stimulation of the tissue it is not possible to gather a parameter that allows for a comparison of the two techniques, but the two outcomes return complementary information. In fact, the force generated by the tissue is a macroscopic parameter, which expresses the contraction of the entire tissue, while the strains obtained through the DIC predictions refer to a specific region of interest. At this point, the strain field measured close to the fixed pin (Figure 5(b)) was observed to entail negative values of the longitudinal strain, with consequent positive values of the transverse strain, in accordance with that obtained during the spontaneous contraction measurements. On the other hand, as hypothesized for isometric electrical stimulations, the strain field obtained focusing on the center of the specimen (Figure 5(c)) is the opposite of that measured at the end of the tissue. Positive values of $\varepsilon_{x}$ were reported and, accordingly, the tissue is locally subjected to a shrinkage, as confirmed by the negative value of the predicted $\varepsilon_{y}$.

Taken altogether, these results show that DIC can be used for accurate and noninvasive measurements of tissue spontaneous contraction frequency; in view of this, the use of DIC can be proposed for repetitive measurements over time to check for tissue maturation. The measurement of the 


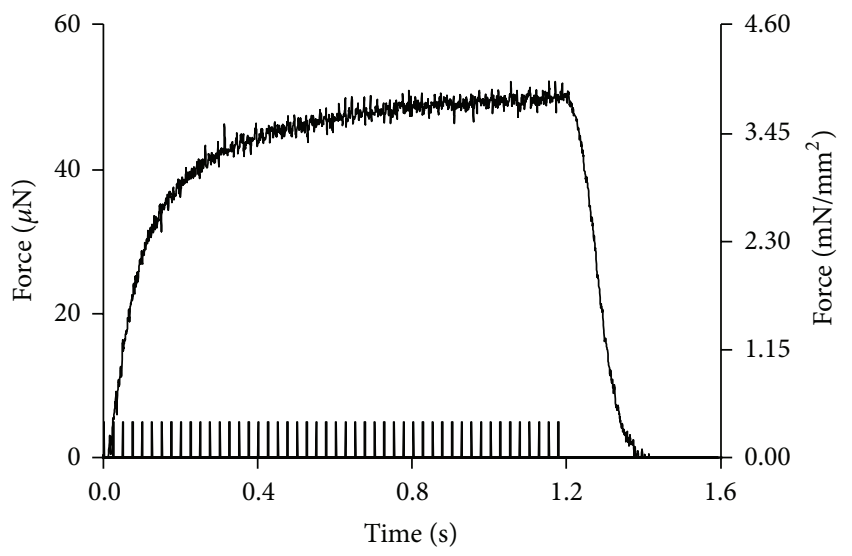

(a)

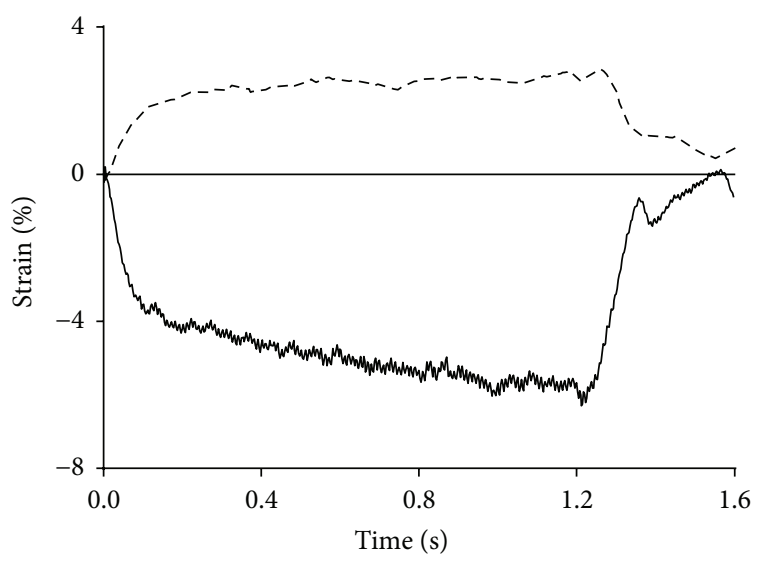

$-\varepsilon_{x}$

(b)

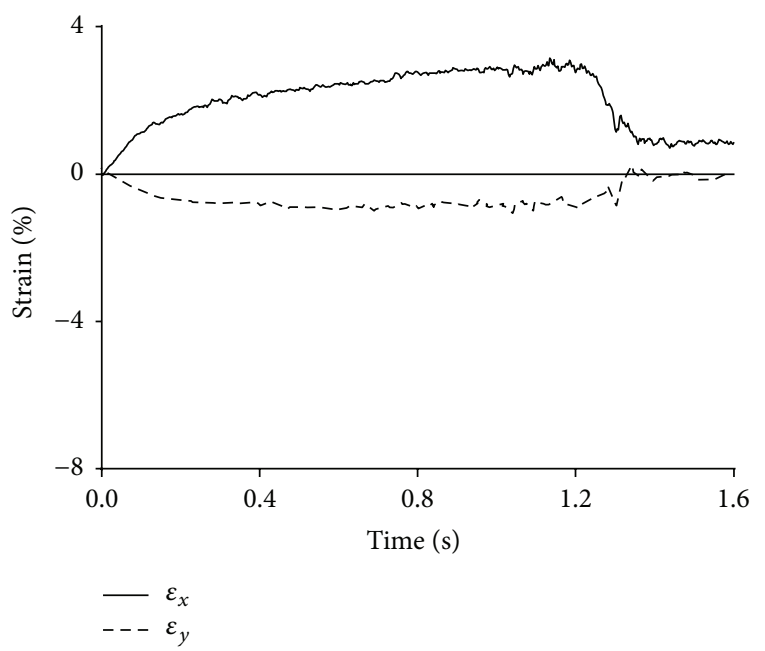

(c)

FIGURE 5: Electrical stimulation. Example of force (a) and strain responses obtained stimulating an X-MET at tetanic frequency and focusing once close to the fixed pin (b) and once on the center (c) of the tissue. Images are acquired at $400 \mathrm{fps}$.

spontaneous contraction frequency by means of the force generated by the tissue, in fact, would imply the specimen to be attached and detached from the transducer each time a measurement is carried out. Moreover, the possibility to measure local strains in a fast, accurate, and noninvasive way could also be of interest to study the time-lapse response of the tissue during drug treatments. A long-term application of this tool could be also the continuous monitoring of tissue growth directly into the incubator, with the possibility to measure its spontaneous contractility at any desired time. On the other hand, the use of DIC as a tool to measure tissue local strains on any desired region of interest during electrical stimulation allows gathering a new parameter to come along with the traditional one, the generated force, to have a comprehensive evaluation of the contractile response of the tissue.

\section{Conclusions}

In this work, we proposed the use of Digital Image Correlation (DIC) as an innovative and noninvasive tool for the measurement of biological tissues' contractile properties. In particular, here we tested this technique to supply accurate values of ex vivo muscle engineered tissue spontaneous contraction frequency. To do this, we compared the frequency values gathered by using the image correlation predictions with the values obtained with the use of a microforce transducer, which represents the standard methodology employed for measuring the contractility of this kind of tissues. In addition to a high speed camera and the force transducer, the experimental system entails a microscope for a proper magnification of the tissue. The use of an optical magnification, together with the very low exposure times needed to follow the high dynamics of the tissue, required a careful balancing between the image resolution and the illumination available for the sensor, to guarantee the proper image contrast for image correlation. Experimental results showed that DIC can be successfully employed as an independent, accurate, and noninvasive tool for the measurement of biological tissue contraction. The relative error values between the spontaneous contractile frequency computed through the predicted longitudinal strain and the force measurement, in fact, were always lower than $0.15 \%$. Moreover, a trend toward even lower relative errors was reported when increasing the acquisition frequency up to $500 \mathrm{fps}$. In view of this, the use of DIC can be proposed for noninvasive measurements of tissue contraction, allowing for repetitive measurements over time to check for its maturation. In addition, DIC can also be used during electrical stimulation of the tissue to gather useful information about the local strain field along with the standard force measurements.

\section{Conflict of Interests}

The authors declare that there is no conflict of interests regarding the publication of this paper. 


\section{Authors' Contribution}

Emanuele Rizzuto and Silvia Carosio equally contributed to this work.

\section{Acknowledgments}

This study was supported by ASI, Fondazione Roma, Telethon (GGP14066).

\section{References}

[1] R. Langer and J. P. Vacanti, “Tissue engineering," Science, vol. 260, no. 5110, pp. 920-926, 1993.

[2] Y. Ikada, "Challenges in tissue engineering," Journal of the Royal Society Interface, vol. 3, no. 10, pp. 589-601, 2006.

[3] S. Carosio, L. Barberi, E. Rizzuto, C. Nicoletti, Z. D. Prete, and A. Musarò, "Generation of eX vivo-vascularized Muscle Engineered Tissue (X-MET)," Scientific Reports, vol. 3, article 1420, 2013.

[4] R. G. Dennis and P. E. Kosnik II, "Excitability and isometric contractile properties of mammalian skeletal muscle constructs engineered in vitro," In Vitro Cellular and Developmental Biology-Animal, vol. 36, no. 5, pp. 327-335, 2000.

[5] R. K. Birla, G. H. Borschel, R. G. Dennis, and D. L. Brown, "Myocardial engineering in vivo: formation and characterization of contractile, vascularized three-dimensional cardiac tissue," Tissue Engineering, vol. 11, no. 5-6, pp. 803-813, 2005.

[6] H. Kondoh, Y. Sawa, S. Miyagawa et al., "Longer preservation of cardiac performance by sheet-shaped myoblast implantation in dilated cardiomyopathic hamsters," Cardiovascular Research, vol. 69, no. 2, pp. 466-475, 2006.

[7] I. A. Memon, Y. Sawa, N. Fukushima et al., "Repair of impaired myocardium by means of implantation of engineered autologous myoblast sheets," Journal of Thoracic and Cardiovascular Surgery, vol. 130, no. 5, pp. 1333-1341, 2005.

[8] E. Rizzuto, S. Carosio, A. Musarò, and Z. Del Prete, "A digital image correlation based technique to control the development of a skeletal muscle engineered tissue by measuring its surface strain field," in Proceedings of the IEEE International Symposium on Medical Measurements and Applications (MeMeA '15), pp. 314-318, Torino, Italy, May 2015.

[9] T. C. Chu, W. F. Ranson, and M. A. Sutton, "Applications of digital-image-correlation techniques to experimental mechanics," Experimental Mechanics, vol. 25, no. 3, pp. 232-244, 1985.

[10] L. Chevalier, S. Calloch, F. Hild, and Y. Marco, "Digital image correlation used to analyze the multiaxial behavior of rubberlike materials," European Journal of Mechanics-A/Solids, vol. 20, no. 2, pp. 169-187, 2001.

[11] M. A. J. Cox, N. J. B. Driessen, C. V. C. Bouten, and F. P. T. Baaijens, "Mechanical characterization of anisotropic planar biological soft tissues using large indentation: a computational feasibility study," Journal of Biomechanical Engineering, vol. 128, no. 3, pp. 428-436, 2006.

[12] G. Okotie, S. Duenwald-Kuehl, H. Kobayashi, M.-J. Wu, and R. Vanderby, "Tendon strain measurements with dynamic ultrasound images: evaluation of digital image correlation," Journal of Biomechanical Engineering, vol. 134, no. 2, Article ID 024504, 2012.
[13] E. Rizzuto, S. Carosio, and Z. Del Prete, "Characterization of a digital image correlation system for dynamic strain measurements of small biological tissues," Experimental Techniques, 2014.

[14] D. J. Williams and I. M. Sebastine, "Tissue engineering and regenerative medicine: manufacturing challenges," IEE Proceedings: Nanobiotechnology, vol. 152, no. 6, pp. 207-210, 2005.

[15] N. Bursac, "Cardiac tissue engineering using stem cells," IEEE Engineering in Medicine and Biology Magazine, vol. 28, no. 2, pp. 80-89, 2009.

[16] M. R. Lewis, "Rhythmical contraction of the skeletal muscle tissue observed in tissue cultures," American Journal of Physiology, vol. 38, pp. 153-161, 1915.

[17] C. Pizza, A. Donoian, S. Booker, and Y. Sun, "Shorteningdeactivation and stretch-activation during spontaneous contraction and relaxation of bivalve cardiac muscles," in Proceedings of the IEEE 32nd Annual Northeast Bioengineering Conference, pp. 23-24, Easton, Pa, USA, April 2006.

[18] Z. Feng, T. Matsumoto, Y. Nomura, and T. Nakamura, "An electro-tensile bioreactor for 3-D culturing of cardiomyocytes," IEEE Engineering in Medicine and Biology Magazine, vol. 24, no. 4, pp. 73-79, 2005.

[19] M. Young, A. Paul, J. Rodda, M. Duxson, and P. Sheard, "Examination of intrafascicular muscle fiber terminations: implications for tension delivery in series-fibered muscles," Journal of Morphology, vol. 245, no. 2, pp. 130-145, 2000.

[20] M. Sciancalepore, R. Afzalov, V. Buzzin, M. Jurdana, P. Lorenzon, and F. Ruzzier, "Intrinsic ionic conductances mediate the spontaneous electrical activity of cultured mouse myotubes," Biochimica et Biophysica Acta-Biomembranes, vol. 1720, no. 12, pp. 117-124, 2005.

[21] E. Bandi, A. Bernareggi, M. Grandolfo et al., "Autocrine activation of nicotinic acetylcholine receptors contributes to $\mathrm{Ca}^{2+}$ spikes in mouse myotubes during myogenesis," Journal of Physiology, vol. 568, no. 1, pp. 171-180, 2005.

[22] L. Madden, M. Juhas, W. E. Kraus, G. A. Truskey, and N. Bursac, "Bioengineered human myobundles mimic clinical responses of skeletal muscle to drugs," eLife, vol. 4, Article ID e04885, 2015.

[23] A. Ahola, A. L. Kiviaho, K. Larsson, M. Honkanen, K. AaltoSetälä, and J. Hyttinen, "Video image-based analysis of single human induced pluripotent stem cell derived cardiomyocyte beating dynamics using digital image correlation," BioMedical Engineering Online, vol. 13, article 39, 2014.

[24] A. Ahola, P. Pradhapan, E. Laurila, K. Aalto-Setala, and J. Hyttinen, "Motion analysis method for determining cardiomyocyte beating properties based on digital image correlation and templates," in Proceedings of the Computing in Cardiology Conference (CinC '14), pp. 1137-1140, Cambridge, Mass, USA, September 2014.

[25] G. Mathews, C. Sondergaard, A. Jeffreys et al., "Computational analysis of contractility in engineered heart tissue," IEEE Transactions on Biomedical Engineering, vol. 59, no. 5, pp. 1429-1435, 2012.

[26] G. B. Broggiato, L. Casarotto, Z. Del Prete, and D. Maccarrone, "Full-field strain rate measurement by white-light speckle image correlation," Strain, vol. 45, no. 4, pp. 364-372, 2009. 


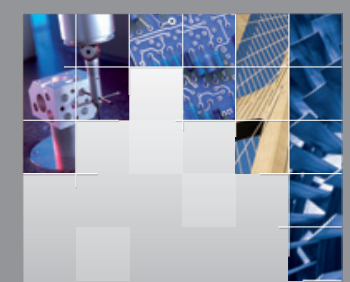

\section{Enfincering}
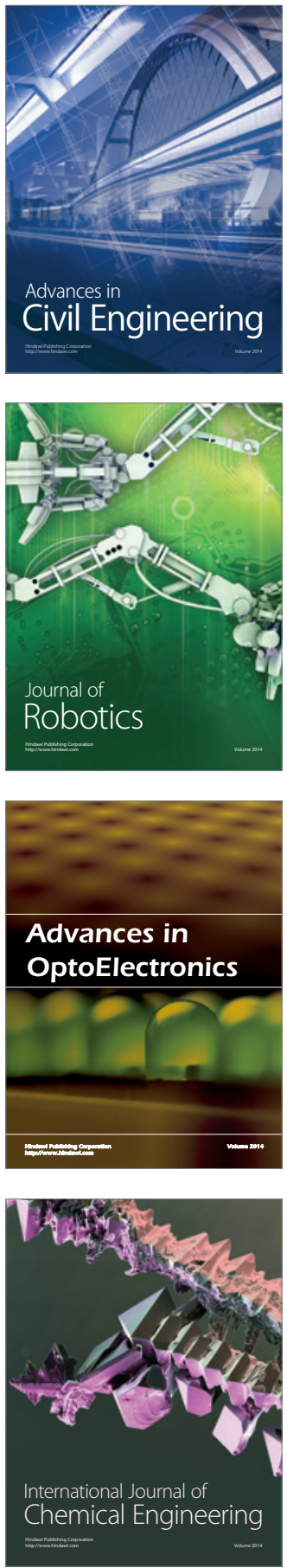

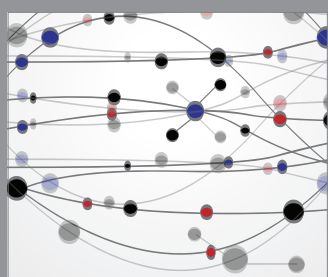

The Scientific World Journal

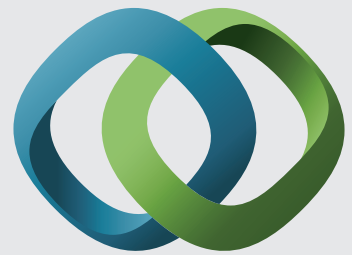

\section{Hindawi}

Submit your manuscripts at

http://www.hindawi.com
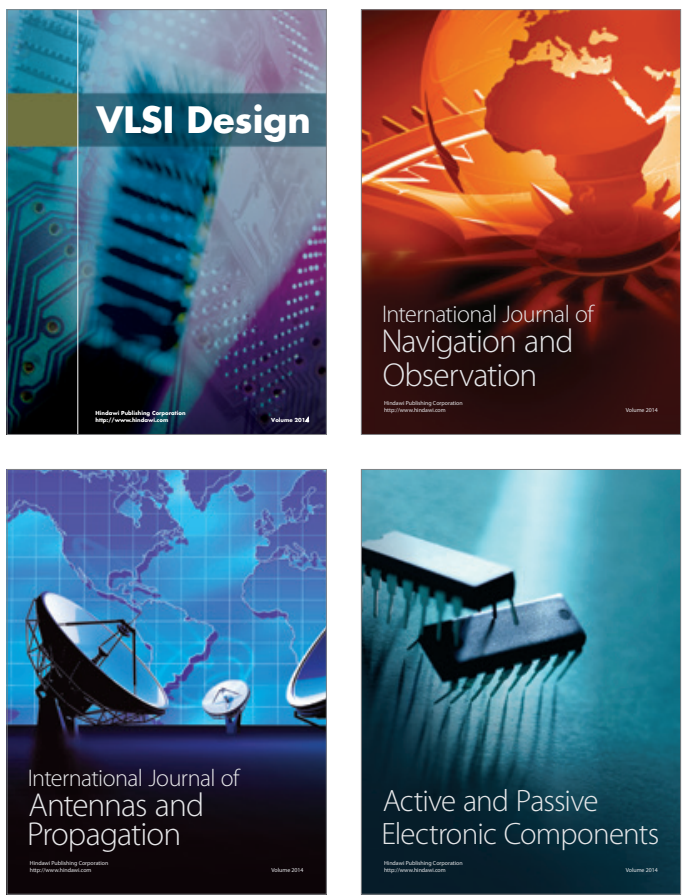
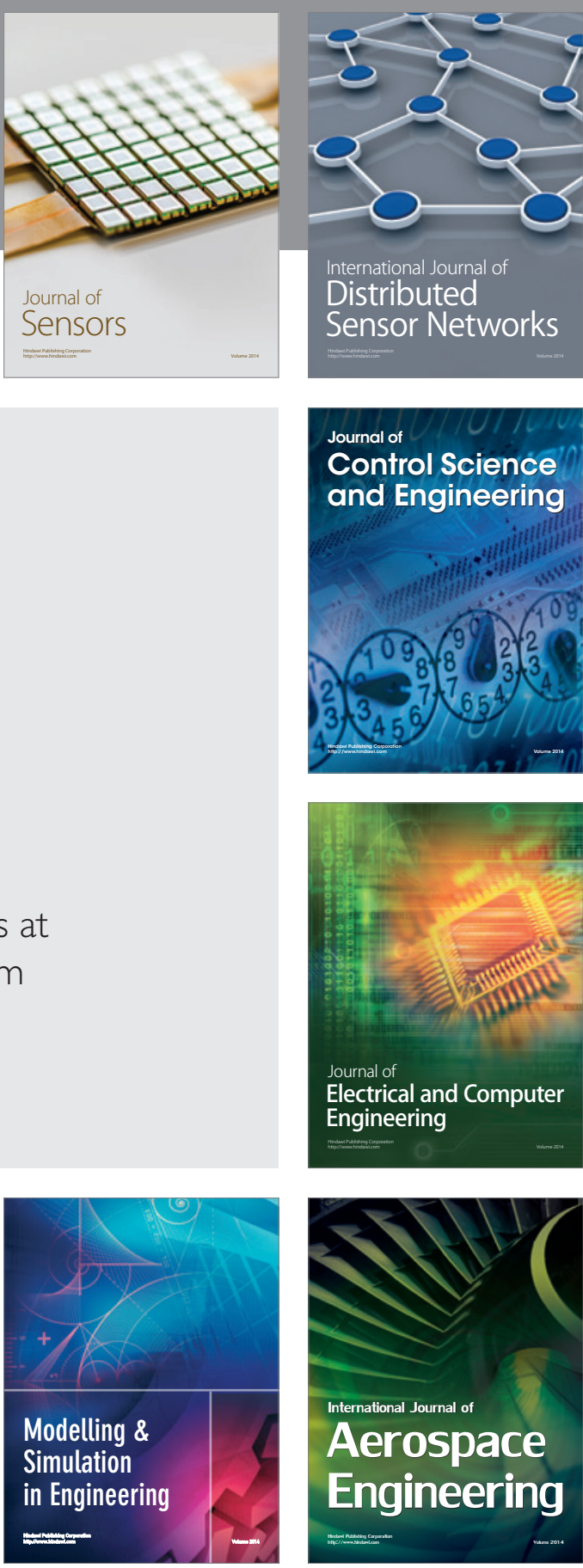

International Journal of

Distributed

Sensor Networks

Journal of

Control Science

and Engineering
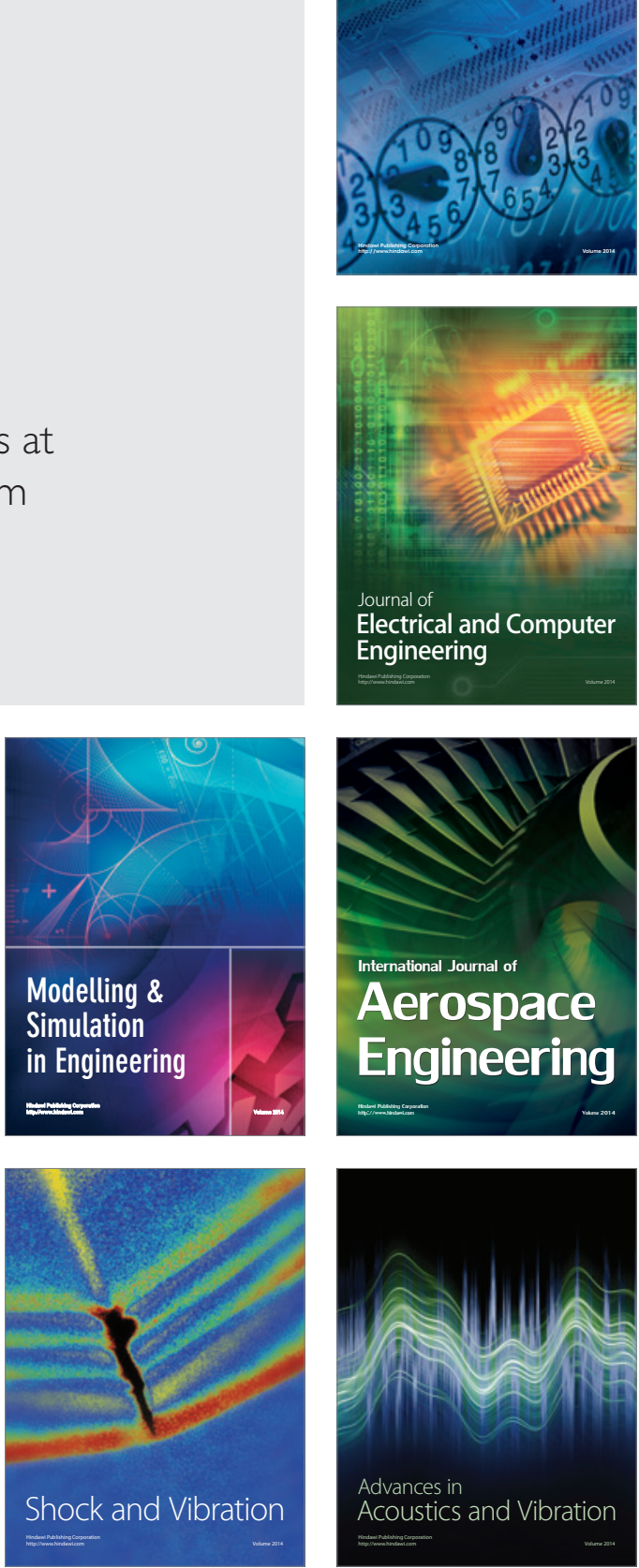